\title{
Finalismo no intencional. Una apropiación aristotélica del vocabulario platónico de la participación
}

\section{MARÍA Elena DÍAZ}

Universidad de Buenos Aires

nosoymary@hotmail.com

Aceptado: 08/06/2018

Recibido: 25/07/2018

Más allá de las críticas de Aristóteles a la noción platónica de participación, existen algunos usos de este término que merecen ser atendidos en la obra aristotélica, en tanto suponen no solo una herencia platónica sino también una resignificación en un plexo conceptual diverso. Este trabajo explora el uso aristotélico de la noción de participación como finalidad no intencional en el argumento que sostiene que uno de los modos de alcanzar la inmortalidad es la procreación, para mostrar cómo se puede compatibilizar la adopción de la terminología platónica de la participación con el entramado causal aristotélico.

Aristóteles / $\mu \varepsilon \dot{\theta} \theta \varepsilon \xi \xi \varsigma$ / Platón / Generación / Causa final

\section{Unintentional Finalism. AN ARISTOTELIAN ApPRopriation of Platonic VocabulaRY OF PARTICIPATION}

Beyond Aristotle's criticisms of the Platonic notion of participation, there are some uses of this term that deserve to be addressed in the Aristotelian work, since they suppose both a Platonic inheritance and a resignification in a diverse conceptual plexus. This paper explores the Aristotelian use of the notion of participation as a non-intentional finality, in the argument that supports that one of the ways of achieving immortality is procreation, to show how the adoption of the Platonic terminology of participation can be compatible with the Aristotelian causal framework.

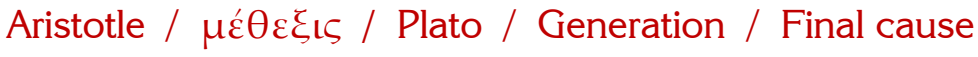


No sin razón se suele considerar el uso del vocabulario de la participación como una marca de platonismo, a pesar de los vaivenes del concepto en el interior de la obra platónica ${ }^{1}$. Menos atención ha recibido de parte de los intérpretes el uso aristotélico de esta noción cuando no está implicado en un cuestionamiento de su maestro. Aristóteles, que realiza un uso muy acotado de este término, se vale en general de la noción de $\mu \varepsilon \varepsilon^{\theta} \varepsilon \xi \iota \varsigma$ para discutir la relación de la especie con el género o, en contextos políticos, para hablar de la relación del hombre con la comunidad ${ }^{2}$. Lo más común es, no obstante, que la participación aparezca mentada en un entorno crítico de la noción platónica ${ }^{3}$. Sin embargo, es posible rastrear en el Corpus un caso especial, próximo a Platón, que supone la apropiación del vocabulario de la participación para dar cuenta de lo que QUARANTOTTO (2005) llama "finalismo no intencional"4. En De anima II 4, 415a25-

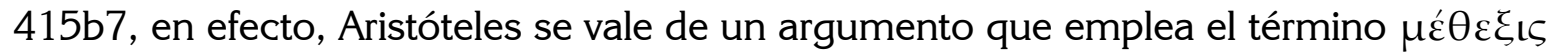
para referirse a la aspiración a la inmortalidad propia de todos los seres vivos, retomando un argumento platónico que, si bien es resignificado en el contexto de la obra aristotélica, conserva tanto el andamiaje conceptual como el vocabulario de su maestro. Se puede advertir, entonces, que Aristóteles no impugna el valor semántico de la noción de participación, que le parece adecuada para caracterizar diferentes tipos de relaciones, lógicas, metafísicas y políticas, sino cómo la entendió Platón respecto de la caracterización de la relación entre las cosas sensibles y las Formas. El filósofo de Estagira, en cambio, coincide con su maestro en que la $\mu \varepsilon \dot{\varepsilon} \theta \varepsilon \xi$ ı cómo los animales tienden, de modo no intencional, a inmortalizarse en la medida de la posible. Solo en el caso de los seres humanos esta tendencia puede volverse deliberada, pero al hacerlo de ese modo excede la mera reproducción y se remonta a otras formas de inmortalidad. La apelación al Symposium platónico es un lugar común en la lista de pasajes relacionados en los comentarios a los textos aristotélicos, si bien no ha sido suficientemente estudiada la naturaleza de la relación (HAMLYN 1968: 95; POLANSKY 2007: 205). Menor es la referencia al argumento análogo de Leges (MOVIA 1979: 298).

En tres lugares del Corpus, De anima II 4, 415a25-415b7, De generatione et corruptione II 10, 336b25ss y De generatione animalium II 1, 731b23-732a1, Aristóteles retoma el argumento platónico de Symposium y Leges que sostiene que los seres vivos pueden alcanzar la inmortalidad por medio de la reproducción. La herencia platónica se advierte tanto en las premisas como en el lenguaje especializado forjado por su maestro a la hora de desplegar el argumento análogo. Aristóteles, por cierto, no se limita meramente a repetir la versión platónica, sino que la adapta a su propio esquema conceptual y a los fines de su argumentación. Es posible advertir, así, cómo un argumento puede actuar de modo proteico, aportando inspiración y andamiaje conceptual, pero a la vez resignificándose. Al reposar sobre diferentes supuestos y entroncarse en un plexo conceptual diverso, en un sentido puede sostenerse que se trata del mismo argumento, y en otro, no. En las versiones platónica y aristotélica se apela a una serie de datos del mundo natural, en el cual está inmerso el hombre, y a partir de esto se 
concluye que opera en todos los animales una tendencia ciega a alcanzar el tipo de inmortalidad que la especie permite, no de un modo individual sino específico, por medio de la cadena de la generación.

La hipótesis defendida en este trabajo es que el uso aristotélico de la noción de participación implica la apropiación de uno de los sentidos de este concepto en la obra platónica: el finalismo no intencional, en un contexto en el cual adopta no solo el concepto, sino el argumento completo.

Comenzaré con las dos apariciones platónicas del argumento en Symposium y Leges, y, a continuación, con la versión de De anima, De generatione et corruptione y De generatione animalium. En Platón, este argumento tiene un rol marginal, en tanto en Symposium solo explica la procreación en las almas y no en los cuerpos, central para el ascenso erótico subsiguiente. En Leges, por su parte, el argumento se edifica sobre bases ético-políticas y no biológicas, en tanto la procreación es identificada como la contribución mínima que deben realizar los ciudadanos a la $\pi$ ó $\lambda ı s:$ darle hijos que permitan la continuidad de la comunidad.

\section{Inmortalizarse por medio de la generación en los diálogos platónicos}

La inmortalidad del alma es uno de los pilares del pensamiento platónico ${ }^{5}$, y puede ser rastreada en muchos de sus diálogos, que la tienen como tema central o recurren a ella para fundamentar otras tesis. Una de sus estrategias de fundamentación de la inmortalidad es el argumento forjado en Symposium y Leges que apela a una tendencia intrínseca a la inmortalidad en el conjunto de todos los seres vivos. Si bien en la filosofía platónica la inmortalidad del alma es una tesis crucial, la contribución de este argumento, como señalé antes, no posee ningún tipo especial de relevancia, que, en cambio, efectivamente tendrá en la obra aristotélica, como expresión del finalismo de los seres vivos. Esto muestra, sin embargo, que quien buscara inspiración en los diálogos platónicos, como lo hizo Aristóteles, para el estudio de un rasgo esencial de la $\varphi v ́ \sigma ı \varsigma$, podía encontrarla.

La versión del Symposium (206c; 207c-208b) se desarrolla en boca de Diotima, al momento de caracterizar a Eros como un impulso fundamental para procrear en lo bello que atraviesa a todos los seres y cumple función de intermediario entre lo humano y lo divino ${ }^{6}$. La maestra de Sócrates sostiene que los animales, humanos y no-humanos pueden inmortalizarse, en la medida en que un cuerpo mortal puede hacerlo, por medio de la generación, por lo cual:

...esta actividad es divina ( $\theta \varepsilon \tilde{c} \mathrm{ov})$, y esto es lo que existe inmortal ( $\dot{\alpha} \theta \dot{\alpha} v \alpha \tau o v)$ en

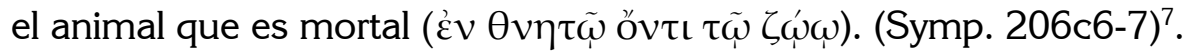

En este pasaje hay un juego de oposiciones, entre lo mortal y lo inmortal, lo divino y lo animal, que se resuelve por medio de la realización, por parte del mortal, de una 
actividad que lo eleva a la inmortalidad y que, como tal, supera la esfera de la vida propiamente animal. Diotima caracteriza a Eros como un ser intermedio, no del todo mortal ni del todo inmortal, y el resultado de su acción en los animales sería también un puente entre lo mortal y lo inmortal ${ }^{8}$. Por ser receptivos a esta acción de Eros, los seres vivos poseen un carácter privilegiado, acentuado en el caso del hombre, pero común a todos los animales. Esta relación entre mortales e inmortales es conceptualizada en el diálogo en términos de participación:

Por este mecanismo, Sócrates, dijo, lo mortal participa ( $\mu \varepsilon \theta \varepsilon \dot{\chi} \chi \iota)$ de la inmortalidad, y el cuerpo y todas las demás cosas; lo inmortal, de otro modo. (Symp. 208b2-5)

El reproche que Aristóteles realiza a sus predecesores al comienzo de De anima9, de haberse ocupado solo del alma humana, no es justo con este pasaje del Symposium que tiene como objeto de reflexión al conjunto de los seres vivos. Es cierto que, al avanzar el diálogo, Sócrates se encarga de reconducir el discurso de Diotima hacia el ámbito propiamente humano, y el resto del discurso erótico se centra solo en sus efectos en los hombres:

Y yo dije, sea así, extranjera, pues dices bien. Siendo así Eros, ctiene alguna utilidad

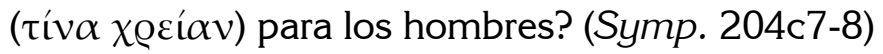

El argumento platónico, ya centrado en la esfera humana, va bastante más allá de la reproducción, en tanto continúa afirmando que por este impulso se desearía tanto la inmortalidad como el bien, pues el amor tiene como objeto la posesión eterna del bien y de la felicidad, y Eros es definido como una tendencia a poseer siempre el bien (206a9). En el discurso de Diotima se contrapone, por otro lado, la procreación corporal a la anímica, destacando la superioridad de la segunda sobre la primera, si bien el deseo que guía a ambas sería idéntico. En el contexto de su exposición esta característica es fundamental, pues Eros es, como ha sido señalado, responsable del carácter unitario y continuo de todo el cosmos ${ }^{10}$.

En el transcurso de este argumento del Symposium se esgrime, a favor del rol de Eros como unificador transversal del cosmos, un conjunto de observaciones que son retomadas por Aristóteles, quien encuentra en ellas una expresión de un fin natural: los animales buscan satisfacer necesidades y obtener placeres inmediatos (comer, beber) y realizan además esfuerzos, e incluso enfrentan dolores y hasta la muerte, para conseguir reproducirse y cuidar a su descendencia. Existen algunos puntos en común entre Symposium 207a-b y uno de los argumentos que Aristóteles despliega en Physica II, 8 a favor del finalismo de la naturaleza entendida como el conjunto de los seres vivos. Allí sostiene que, en el caso de las plantas, la hoja se genera en vistas del fruto y la raíz 
en vistas de la nutrición. Del mismo modo, la golondrina hace el nido y la araña la tela, en un caso para la reproducción y en otro para la conservación (Ph. II 8, 199a20-30). En ambos textos, pues, las conductas animales que tienden sistemáticamente hacia la reproducción son interpretadas como expresiones de una tendencia a traspasar los límites de la vida caduca del individuo. En el caso de Banquete, por un impulso a la inmortalidad que atraviesa todo el cosmos, mientras que el texto aristotélico busca probar que la forma de las entidades naturales es también causa final (Ph. II 8, 199a3132). Solo en el pasaje de De anima II 4 que consideraremos más adelante, Aristóteles establece una relación explícita entre las entidades naturales y la tendencia a la inmortalidad, lo cual estrecha la relación con el texto platónico.

En ambos filósofos, entonces, se utiliza el comportamiento de los animales irracionales respecto de la reproducción en apoyo de un argumento que sostiene una organización causal final. El voũ s ordenador que Sócrates lamentaba no haber encontrado cumpliendo un rol efectivo en el planteo de Anaxágoras, en el célebre pasaje anterior a la segunda navegación del Phedon (96a-102a), opera en las versiones platónica y aristotélica del argumento como una tendencia inmanente a la inmortalidad que excede la barrera humana y sostiene el finalismo del conjunto de los seres vivos. Platón, en Symposium, extiende esta organización al conjunto del cosmos. Más adelante en este trabajo discutiré si esta estructura final, en el caso de Aristóteles, puede extenderse más allá del límite de la naturaleza de animales y plantas.

El paralelismo entre Physica II, 8 y el pasaje mencionado del Phedon es tal que Aristóteles coincide, además, con Platón, en criticar a quienes no han dado cuenta de modo efectivo del rol de la causa final a pesar de haber propuesto un principio inteligente ordenador. Estos pensadores, según Aristóteles, "si mencionan otra causa, el amor, el odio, el intelecto, se despiden de ella apenas haberla asido" (198b13-16). En Phedon 98b, Sócrates se había lamentado de que Anaxágoras, a pesar de proponer al voũs como principio, no le había atribuido ningún rol efectivo en el ordenamiento de las causas. Platón y Aristóteles encontraron un modo de explicar el funcionamiento de la naturaleza de modo finalista, mostrando no solo que hay un orden inteligente e inteligible que rige el cosmos, sino asumiendo el desafío de dar cuenta de su efectiva operación con algunos ejemplos específicos. Los textos coincidentes de Symposium y Physica que acabamos de ver son una prueba cabal de esta estrategia argumentativa que consiste en concluir el finalismo natural teniendo como premisas el comportamiento ordenado de los animales irracionales.

En Leges IV, 721b-d una línea argumentativa similar a la del Symposium es esgrimida por Platón como parte del preámbulo de la ley acerca del matrimonio, y por medio de ella se intenta persuadir a los ciudadanos para que se casen entre los treinta y los treinta y cinco años y tengan hijos. Para fundamentar las ventajas del matrimonio se recurre a una premisa sostenida como un axioma: por naturaleza se desea alcanzar la inmortalidad por todos los medios (721b7-c1). La forma más común que adquiere este impulso, el deseo de fama más allá de la muerte, es entendida en este argumento como 
una manifestación de esta tendencia originaria. Según la ley propuesta, en tanto una de las formas por medio de las cuales la raza humana logra la inmortalidad es la pro-

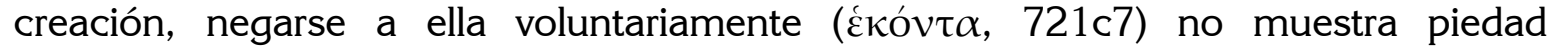

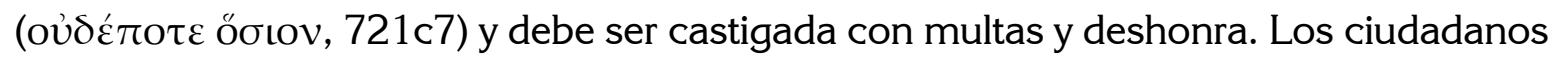
más jóvenes, en efecto, que tienen que ser respetuosos con los mayores, no deberían guardar la misma consideración con aquellos que no hubieran proporcionado hijos a su ciudad. En la fundamentación se mezclan dos planos: el político, en tanto la procreación es considerada como un servicio que los ciudadanos prestan a su ciudad, y el religioso, dado que negarse voluntariamente a la inmortalidad es una ofensa contra los dioses $^{11}$. Esto último se explica solo por la derivación de la procreación del axioma de la tendencia a la inmortalidad, lo cual lo vincula con la versión del Symposium ${ }^{12}$. Sin embargo, al centrarse en su aspecto político, la versión de Leges deja de lado las distinciones del Symposium acerca de la posibilidad de expresar este afán por medio de la procreación en las almas, y no por medio del matrimonio. En su comentario a este pasaje de Leges IV, Christopher RowE (2010: 49-50) sostiene que la premisa platónica de que todo deseo tiene como fin la inmortalidad solo puede entenderse a la luz de la versión del Symposium que explica cómo se verifica este deseo en planos que exceden a la mera reproducción biológica.

En el argumento de Leges, los forjadores del código legislativo admiten que, a diferencia del resto de los animales, el hombre puede negarse a la reproducción, o bien realizarla de un modo perjudicial para la comunidad política. Actualizar esta capacidad de su naturaleza sería, para los seres humanos, una elección, a diferencia de lo que ocurre con los animales irracionales. En esto también Aristóteles se revela como heredero de Platón, en tanto en su aspecto práctico, la naturaleza racional del hombre opera como un conjunto de disposiciones que tienen que ser actualizadas por medio de la educación, los buenos hábitos y la decisión racional ${ }^{13}$. Se puede sostener, con LLOYD (1993), que hay una dimensión normativa en este rasgo de la naturaleza humana. ${ }^{14}$ Como no se trata de una necesidad compulsiva que mueve solo en una dirección prefijada, la naturaleza humana es susceptible de corrupción. Esto implica que la tendencia a la inmortalidad, que permite al hombre participar de lo divino, cristalizada en la búsqueda de fama o de dejar descendencia, puede conducir al hombre al error y al vicio. Por tanto, el legislador debe arbitrar medidas para que esos impulsos naturales se cana-

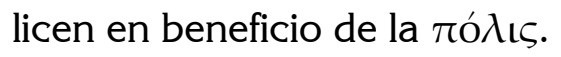

Tanto en Symposium como en Leges, la unión sexual y la búsqueda de fama antes y después de la muerte son interpretadas como manifestaciones del deseo de inmortalidad propio de todos los seres vivos. En ambos diálogos, en efecto, la perspectiva desde la cual se argumenta es la del género de los seres vivos o la especie humana en particular, pero en función de una característica común al resto de los animales. Solo los hombres podrían, sin embargo, entender la naturaleza del impulso que lleva a la procreación, tanto en el plano cósmico como político. En Symposium la tendencia a 
la procreación es el resultado de la acción de Eros, que sirve de este modo como intermediario entre lo mortal y lo inmortal. En el argumento de Leges, se acentúa que la procreación es lo que permite el desarrollo del género humano a lo largo del tiempo, y así otorga una base biológica a las consideraciones políticas y religiosas que constituyen el núcleo duro del preámbulo persuasivo. El argumento platónico desplegado en ambos diálogos encontró eco en el pensamiento aristotélico, como recurso para sostener el tipo de inmortalidad que son capaces de alcanzar las oủoí $\alpha \iota$ naturales.

\section{Apropiaciones aristotélicas del argumento platónico}

He mostrado al comienzo de este trabajo que los usos aristotélicos del vocabulario de la participación son limitados. Si dejamos de lado los casos en los cuales Aristóteles está criticando la noción platónica, podemos agrupar en tres los usos dentro de sus desarrollos teóricos: en el campo de la política, respecto de los individuos y la comunidad; en la esfera lógica, para remitir a la relación de las especies con los géneros o los individuos o las especies con sus características propias; $y$, por último, aquel que forma parte del eje central de este trabajo, dentro del campo psicológico, que afirma la tendencia del alma a la inmortalidad.

A la hora de tematizar el tipo de inmortalidad que son capaces de alcanzar las ousíai naturales, Aristóteles sostiene la existencia de una tendencia de todos los seres vivos a inmortalizarse en la medida de lo posible. A primera vista, esta línea argumental no parece problemática y encaja perfectamente con los escritos biológicos del estagirita: cada tipo de ser aspira a la inmortalidad y la actualiza en la medida de las potencialidades de su especie. Los seres vivos, que no son capaces de gozarla de modo individual, pueden, de todos modos, alcanzarla dejando tras de sí un individuo poseedor de idéntica forma sustancial. Sin embargo, una atenta mirada a las diferentes apariciones de este argumento en el Corpus deja abierta una serie de interrogantes sobre cuál es el motor de esta tendencia a la inmortalidad y cómo actúa en cada especie, incluyendo la humana, que dispone, además, de otros mecanismos de trascendencia que exceden la reproducción biológica ${ }^{15}$. El disenso entre los intérpretes acerca de qué moviliza esa aspiración de trascendencia muestra un abanico de posibilidades. Más allá de los matices que veremos más adelante, el motor se ha considerado como una tendencia inmanente a los seres vivos o bien como un impulso hacia una entidad trascendente ${ }^{16}$. En mi argumentación mostraré que ambas interpretaciones son compatibles y pueden ser integradas en una explicación más abarcadora. Además, sostengo que en la interpretación de la argumentación aristotélica no se debe dejar de lado la influencia de las versiones platónica del argumento, en tanto se trata de un caso de excepcional proximidad conceptual y terminológica.

En el contexto de la indagación del finalismo natural expresado en la diferenciación sexual, Aristóteles se vale, en De generatione animalium II 1, 731b23-732a1, del argu- 
mento platónico de la sección anterior, adaptándolo a los fines de sus propias necesidades teóricas ${ }^{17}$. Una de las premisas de la versión aristotélica sostiene que vivir es mejor que no vivir ${ }^{18}$ y que este es el motor que impulsa a los animales a la reproducción. El tipo de ser característico de animales y plantas, en efecto, al ser contingente, lleva en sí mismo la posibilidad de su no existencia. La reproducción sería un modo de superar este límite, no numéricamente sino de modo específico. El argumento prosigue mostrando por qué es mejor, para los fines de la reproducción, que macho y hembra estén separados, en función de la aplicación de los pares materia y forma, potencia y acto como como complementarios a partir de la especificidad de la función propia de cada uno. La forma y el acto serían siempre prioritarios, y esta jerarquía no sería característica solo de la diferenciación sexual, sino que estaría también presente en otros casos. Aristóteles divide allí, en efecto, las cosas que son en dos tipos bien diferenciados e interrelacionados: las divinas, que son eternas y bellas, y las contingentes, y afirma que las primeras son causas de las segundas. Despliega esta duplicidad jerárquica en pares de instancias complementarias: el alma es mejor que el cuerpo, la forma que la materia, lo animado que lo inanimado, el ser que el no ser, el vivir que el no vivir, para desembocar finalmente en que el macho es mejor que la hembra, dado que lo asocia al principio activo ${ }^{19}$. Así, la prioridad de la forma aparece como la expresión de lo eterno y divino en el hombre, que tiene la capacidad de transmitirse, por medio del macho, a la hembra que es entendida únicamente como la que aporta la materia en el proceso de generación. Sin embargo, esta separación y jerarquización es atenuada por Aristóteles en términos de complementariedad. La forma sola no puede dar lugar a la generación, y por eso afirma que "la reproducción es común a ambos" (732a10). Esto permite al individuo alcanzar el máximo tipo de eternidad que le es posible y garantiza la eternidad de la forma. De aquí deriva la importancia que Aristóteles le otorga a la génesis $\alpha \pi \lambda \tilde{\omega} \varsigma$ en Physica, como base para el carácter privilegiado de la forma en la filosofía primera ${ }^{20}$. En Physica V Aristóteles refina la terminología del movimiento para puntualizar la diferencia entre la generación y otros tipos de movimiento que suponen como sustrato al compuesto. Este tipo de generación, considerada en este último texto

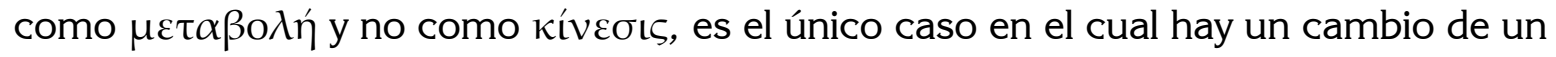
no-sujeto a un sujeto, de allí su caracterización de " $\alpha \pi \lambda \omega \tilde{\omega} \varsigma$ ", propiamente dicha o absoluta (225a12-17). Es decir que la generación de una entidad es un caso especial, que no se deja conceptualizar como movimiento, en tanto no se da entre contrarios (tal como se caracteriza el movimiento en Ph. I 7, 190b10-17 y Metaph. X 4, 1055b11-17).

Sobre la base de lo argumentado hasta aquí, estamos en mejor posición de evaluar el uso del vocabulario de la participación en una variante del argumento anterior. Se trata de De anima II 4, cuando Aristóteles despliega las funciones de la facultad nutritiva del alma, potencia primera común a animales y plantas, y le adjudica, además de la alimentación, la reproducción ${ }^{21}$ que califica de "la más natural de las obras"

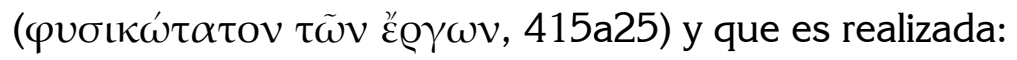


para participar ( $\mu \varepsilon \theta \varepsilon \dot{\varepsilon} \chi \omega \sigma \mathrm{t} v)$ de lo eterno y lo divino en cuanto les sea posible; todos, en efecto, desean (ỏ@é $\gamma \varepsilon \tau \alpha \mathrm{l}$ ) aquello y a causa de aquello hacen cuanto

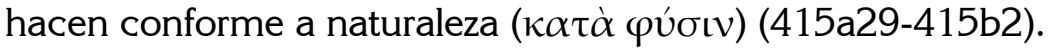

Este pasaje es central para entender este uso aristotélico positivo y original de la noción de participación. Unas líneas más abajo, aparece dos veces, además del verbo

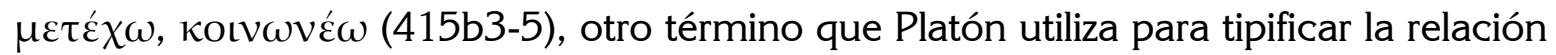
entre las Ideas y las cosas ${ }^{22}$. Aristóteles está, entonces, apelando a un argumento platónico y lo hace, además, valiéndose de la terminología técnica de su maestro. Sin embargo, tanto los términos como el argumento cobran en la obra aristotélica un sentido diferente al de los diálogos que emula, como veremos a continuación. Este juego de convergencias y divergencias ya fue destacado en el comentario de HAMLYN (1993: 95), quien advertía acerca de que ni los términos ni el argumento poseen el mismo sentido en ambos filósofos ${ }^{23}$. El comentarista sostiene que la diferencia entre la doctrina sostenida por Diotima en Symposium y la versión aristotélica reside en que para este último se trata de un proceso natural y que lo que está en juego es la preservación de la especie. Me siento inclinada a pensar, sin embargo, que la diferencia no radica en este punto, dado que, en Symposium, según he mostrado, también se alude al proceso natural de la reproducción de la vida que atraviesa todas las especies. El hecho de que luego en este argumento se produzca un viraje decisivo al sentido no biológico no quita el matiz biológico de la apreciación de la cual parte. Al avanzar en mi argumentación mostraré que la diferencia radica más bien en la concepción de lo divino de cada filósofo, lo cual conlleva también el tipo de relación causal que se verifica entre el dios y el resto del cosmos.

Antes de ir a la interpretación del pasaje, despejaré una alternativa de lectura que no considero viable, y que, si bien ha recibido duras críticas, permanece todavía en el horizonte de sentido de los intérpretes de Aristóteles, en gran parte porque dio un nuevo enfoque e impulso al estudio de su obra. Más allá de que en ciertos casos la solución evolutiva pueda ser una vía legítima, no creo que sea fructífero aquí aducir un pasaje de redacción temprana dentro de un periplo que alejaría a Aristóteles paulatinamente del platonismo, como ha aducido JAEGER (1923: 58-59). La adopción de terminología platónica a la hora de apropiarse de uno de sus argumentos indica que la crítica de Aristóteles a la noción de participación se refiere más a su aplicación a la relación entre cosas e Ideas que a considerarlo un término inaceptable desde el punto de vista filosófico. En otras palabras, el problema no consiste, para Aristóteles, en el uso del vocabulario asociado a la participación, sino en la explicación de las cosas sensibles por medio de la postulación de principios que pertenecen a otro ámbito.

En el medio del pasaje de De anima que estamos considerando, con una breve aclaración parentética, Aristóteles indica, tal como lo hace en otras obras, que el tér-

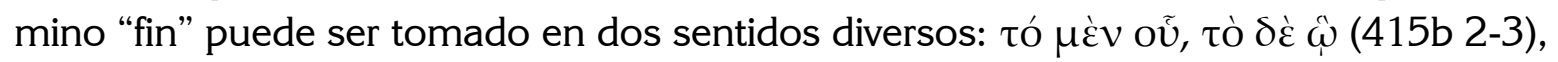


"el fin mismo de algo" y "aquello para lo cual esto es un fin" -en términos de LEFÈVRE (1972: 142), el "beneficiario"24 . El pasaje permite múltiples lecturas tanto respecto de qué constituye cada sentido de fin como respecto de cuál es el propósito de la aclaración aristotélica. La interpretación más clásica es la de RODIER (1900, seguido por Ross 1961), quien entiende que el primer fin "eterno y divino" es el primer motor y, el segundo, los seres naturales, especialmente los animales. Según LEFÈVRE (1972: 142-4), se trata de los mismos seres naturales en ambos fines, que, por un lado, tienden a una divinidad que les es negada como individuos y, por otro, concurren a construir un orden divino y eterno que es tanto lo que funciona como fin como el beneficiario del fin. MOVIA (1979; 2001: 270) entiende, con Ross, que Aristóteles se está refiriendo solo al fin en el primero de los sentidos indicados, aunque aclara que no se trata más que de la eternidad de la especie, respecto de la cual el alma actúa como causa motriz. SEDLEY (1991) propone una lectura antropocentrista del finalismo aristotélico y entiende que el beneficiario de la reproducción de plantas y animales sería el hombre, sin que esto excluya que cada especie tiene como fin inmediato la perpetuación de la forma, más allá del carácter perecedero de los individuos compuestos. El fin de la naturaleza entendida como el conjunto de los seres vivos, sería, sin embargo, el hombre y, a nivel cósmico, el dios (SEDLEY 1991: 192 y 196). GERSON (2005: 123-124) considera que está fuera de discusión que el segundo de los sentidos se corresponde con los seres vivientes, mientras que el primero puede ser dios, los dioses y lo que sea que funcione como principio de la naturaleza, y que la cuestión desafiante para el intérprete es ver cómo se relacionan ambos sentidos. Según mi lectura, existe una cadena de fines, tal como puede leerse, por ejemplo, en Politica I 8, 1256b15-22, que desemboca en el hombre, pero sigo a autores como BURNYEAT (2004) y FALCON (2005) y la restrinjo al ámbito sublunar, que es el involucrado en este pasaje ${ }^{25}$. El pasaje de Política, sin embargo, no impide la identificación de fines internos a cada especie, que procura su maduración y reproducción, sino que establece cómo cada especie puede servirse de otras para cumplir su fin ${ }^{26}$. Para JUDSON (2005), todo lo que ocurre de modo regular en el ámbito sublunar no tiene como fin la supervivencia humana, sino la prosperidad de los seres vivos, en tanto las especies tienden a mantenerse en la existencia y reproducirse, incluso aquellas que no están involucradas en ninguna cadena que desemboque en el hombre. Entonces, teniendo en cuenta el pasaje de Politica que acabo de mencionar y las advertencias de JuDSON (2005) y Rossi (2010), se puede pensar que Aristóteles entiende que los individuos de cada especie tienden a prosperar y reproducirse, y que para ello se valen de otras especies, pero esto no significa que exista un fin exterior, esto es, que el cereal crezca para alimentar al hombre. La capacidad humana racional plasmada en el desarrollo de la técnica hace que sea más eficaz en el aprovechamiento de otras especies para sus fines, pero eso no significa que el árbol crezca para que el hombre pueda realizar muebles, sino que el hombre se vale de todos los recursos como si estuvieran ahí para él ${ }^{27}$. El argumento de De anima II 4 acerca de la tendencia a inmortalizarse en la medida de lo posible invita a entender, además, cómo 
opera la relación causal defendida por Aristóteles más allá de los límites de la existencia humana.

Creo que la clave de interpretación del argumento aristotélico objeto de este trabajo radica en las numerosas ocasiones en las cuales Aristóteles vincula la tendencia a la inmortalidad de la especie con el movimiento circular eterno de las esferas celestes. No porque, como mostraré a continuación, sean estas el fin del movimiento, sino porque da la pauta de que esta tendencia no puede explicarse solo en términos de una característica inmanente de las oúoí $\alpha \iota$ naturales, sino en función del todo ordenado del cual estas forman parte. En un sentido físico, el movimiento incesante de la esfera celeste del sol posibilita el ciclo de la vida, tal como Aristóteles afirma en De generatione et corruptione II, 10. En este texto, Aristóteles afirma, tal como lo habíamos visto en la aparición de nuestro argumento en De generatione animalium, que es mejor ser que no ser. Y como toda la naturaleza tiende hacia lo mejor, todo lo natural tiene como fin permanecer en el ser tanto como sea posible para las potencialidades de la especie. Esta tendencia la cumplen de diferente modo los distintos tipos de oúoí $\alpha$. La oủoí $\alpha$ suprasensible eterna y la sensible eterna lo hacen de un modo numérico, mientras que, como vimos, la oủoí $\alpha$ sensible corruptible solo es eterna por su forma específica, pero no de modo numérico. Los cuerpos simples, dice Aristóteles, imitan, por medio de la generación y la corrupción incesante, este movimiento circular. También en este texto de De generatione et corruptione la tendencia a la eternidad es reconducida por Aristóteles a la actuación de un principio divino (ó $\theta \varepsilon o ́ s, 336 b 32$ ). Otra vez se encuentran, en un argumento que trata la tendencia a la inmortalidad, ecos de los diálogos platónicos: el papel de la divinidad y la $\mu$ í $\eta \sigma \iota \varsigma$, en este caso, en lugar de la participación

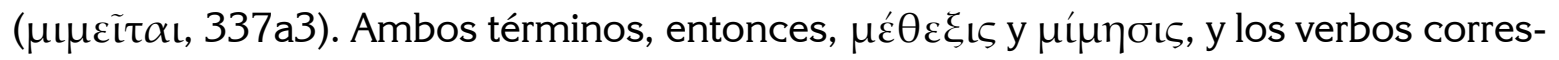
pondientes, serían recursos por medio de los cuales Aristóteles expresa el finalismo no intencional que da cuenta de gran parte del orden cósmico. Precisaremos a continuación los lineamientos generales de este finalismo en el conjunto de la obra aristotélica.

En la filosofía aristotélica el armazón causal es complejo y ambicioso. Si apelamos a Metaphysica XII, vemos cómo Aristóteles explica el movimiento de las oủoí $\alpha$ ı naturales apelando a una cadena causal eficiente que opera a nivel próximo o remoto: en el caso de los animales, el próximo es el padre; el remoto, el movimiento de la eclíptica ${ }^{28}$. Pero esta causa remota del movimiento, que es eterna, tiene a su vez otra causa, también eterna, de su movimiento en la esfera celeste contigua, de modo tal que, para no caer en el infinito, Aristóteles termina postulando un primer motor, causa eterna inmóvil. De este modo, el fin al que Aristóteles se refiere como eterno y divino en el pasaje de De anima remite, según mi lectura, a la eternidad de la forma. El estudio de cómo, en otro nivel causal, la eternidad de la forma depende, además, del movimiento de las esferas celestes que a su vez dependen del o de los motores inmóviles, y especialmente el primero de ellos, no es ya objeto de estudio de un tratado de psicología sino de la filosofía primera entendida como teología. ${ }^{29}$ La controversia acerca de si el primer motor actúa solo como causa eficiente, final o ambas se remonta a la Antigüedad y sigue 
estando vigente, tal como muestra Ross (2016), quien recientemente volvió sobre la defensa de la causalidad final. En su argumento a favor de esta posición tradicional, Ross aduce el pasaje de De anima II 4 acerca de la tendencia hacia la inmortalidad y entiende que se puede interpretar lo que ya había sido identificado por BROADIE (1993) como "causa ejemplar" en términos de causa final. Para Ross (2016: 214), en pasajes como los analizados de modo central en este trabajo, Aristóteles estaría sosteniendo dos tipos de causa final: una intrínseca, propia de cada especie y otra extrínseca por la cual se ordena respecto del cosmos. Según lo que sostuve antes, no se trata del mismo nivel explicativo en ambos casos, por lo que difícilmente se puedan clasificar como el mismo tipo de causa. Solo lo que Ross denomina "causa intrínseca" opera realmente como causa $^{30}$, mientras que lo que él entiende como "causa extrínseca" alude a aquello sin lo cual la causa intrínseca no podría darse. En la explicación de la generación, es necesario, como vimos antes, el movimiento del sol y este, a su vez, forma parte de un entramado astronómico sustentado por una base metafísica ${ }^{31}$.

Los movimientos sublunares no pueden poseer la eternidad propia de los supralunares, que son numéricamente eternos, sino que reflejan la eternidad en la medida en que los seres corruptibles pueden hacerlo: por medio de la continuidad de la especie, y esto es precisamente lo que Aristóteles aclara en el pasaje de De anima que nos ocupó antes, en el cual afirma que animales y plantas participan de la eternidad en la medida de lo posible. Esta medida de lo posible está condicionada por la materia ${ }^{32}$ y la composición de los cuerpos, pues el movimiento circular eterno de los astros es posible gracias al éter y la inmovilidad del primer motor en virtud de la ausencia de materia. La garantía de la continuidad de la especie no puede depender solo de los seres que pertenecen a ella. Como todos los individuos de una especie son, a nivel individual, corruptibles, la eternidad de la especie requiere como causa una entidad eterna ${ }^{33}$. Para entender el argumento sobre la tendencia a la inmortalidad implicada en la generación, se debe tener en cuenta que los tres tipos de entidades desempeñan un rol, pero el más pertinente es, por cierto, el de la forma inmanente de las entidades naturales. El periplo explicativo aristotélico suele partir de la constatación de que, por ejemplo, hombres engendran hombres, para remontarse a la forma, el movimiento de la eclíptica y el primer motor inmóvil. Esto es así porque el camino inverso sería inaceptable, en tanto de la inmovilidad del primer motor no se deduce el movimiento de las oúoí $\alpha$ ı natura$\operatorname{les}^{34}$. Esto nos conduce a un detalle importante del argumento de De anima II 4, objeto de mi indagación. Más allá de la coincidencia con el texto platónico de Symposium acerca de la expresión de algo divino en la tendencia a la inmortalidad, existen diferencias destacables en ambos filósofos en cuanto a la concepción de la divinidad. BOTTER

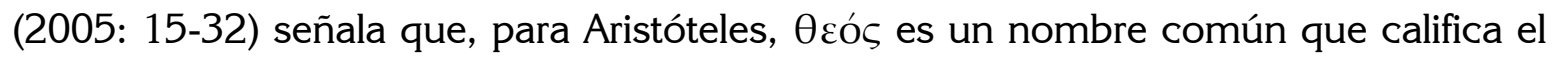
grado supremo de una escala jerárquica, por lo que le corresponde, no de modo exclusivo, pero sí principal, al primer motor inmóvil postulado para explicar la eternidad del movimiento. Aristóteles califica de divinos a los astros y a los motores que los mueven, además de usarlo para la actividad del intelecto. En los diálogos platónicos, el dios 
desempeña una función diferente, en tanto no es postulado para dar cuenta del movimiento y posee una connotación moral, dado que se constituye como un paradigma al que el hombre debe intentar asimilarse (Theaetetus 176a8-b3; Timaeus 90b6-90d8; Respublica VI, 500c9-d2; Leges IV, 716c1-d4). Cuando Aristóteles se vale de la imitación, como vimos, no lo hace en calidad de paradigma moral sino en función de la eternidad.

En el pasaje de Physica II, 8 que mencionamos antes, Aristóteles, como antes Platón en el Symposium, se vale del caso de los animales no humanos para probar la naturaleza finalista de los seres vivos, que no depende de la voluntad de los agentes. Poseer una forma sustancial trae consigo una tendencia a la inmortalidad que es opaca para la mayoría de los agentes. Esto es así porque, para Aristóteles, la forma no se genera, y, a diferencia de otras instancias que tampoco se generan, como la materia, para poder adquirirla se requiere de un animal de la misma especie que la posea en acto y la transmita por el proceso de generación (Metaph. VII 9, 1034b8-19). Algunos hombres, igual que el resto de los animales, expresarían solo de este modo esta aspiración, esto es, sin tener plena conciencia del sentido último de una acción tan básica y natural como la reproducción. Se centrarían únicamente en el placer sexual o en el deseo de perpetuar su nombre más allá del límite de la muerte personal, siendo recordado y honrado por sus descendientes. Pero, para otros hombres, entender que la generación obedece a un fin sería el comienzo del camino para comprender el eterno orden inteligible del cosmos, lo más alto que un hombre puede alcanzar a nivel teorético. Entonces, así como en el argumento platónico del Symposium hay una apertura para formas de inmortalidad más excelsas que la reproducción, también esto está presente en el pensamiento aristotélico, si bien para rastrearlo hay que recurrir a los escritos prácticos. Lo que sirve de nexo entre las versiones aristotélicas del argumento acerca de la posibilidad de alcanzar la inmortalidad por medio de la reproducción y aquellos pasajes que ensalzan la superioridad de la vida teorética es la remisión a lo divino. En definitiva, se trata de la misma tendencia hacia la inmortalidad, expresada conforme a las posibilidades de la especie humana.

Me voy a valer de un texto de Ethica Nicomachea VII, 13 para ilustrar cómo Aristóteles entiende que opera la tendencia hacia lo divino en la mayoría de los hombres. La tendencia a la inmortalidad, tanto en las versiones platónica como aristotélica del argumento, suele ser opaca para los agentes, esto es, no supone, necesariamente, un propósito deliberado de trascendencia hacia lo divino. En su primer tratamiento del placer en esta obra, que tendrá su complemento en la discusión del Libro X, Aristóteles señala que, dadas la universalidad y la atracción de los placeres corporales, la mayoría de los hombres creen que son los únicos que existen. Si bien no está de acuerdo con esto último, entiende que la universalidad del placer es algo digno de ser tenido en cuenta. Después de llamar la atención acerca de que tanto los hombres como los animales persiguen el placer, ofrece esta razón para justificar de qué modo tendría sentido afirmar que el placer es el bien supremo: 
Sin embargo, como la misma naturaleza ni es ni parece ser la mejor disposición, no todos persiguen el mismo placer, aunque sí todos persiguen el placer. Quizás persiguen no lo que creen ni lo que dirían, sino el mismo, pues todas las cosas,

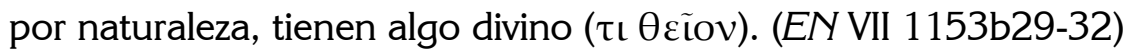

En su comentario a este texto, GAUTHIER Y JOLIF (1970: 810) llaman la atención acerca de los tres planos de análisis que Aristóteles distingue en este pasaje: el plano de la apariencia, el plano de la realidad superficial y el plano de la realidad profunda. En el plano de la apariencia, los placeres corporales son los mejores o bien los únicos que existen. En el plano de la realidad superficial, todos los hombres gozan de los placeres corporales; en el de la realidad profunda, esta inclinación a disfrutar del placer es interpretada como una tendencia natural hacia lo divino. Es solo bajo esta última consideración que puede sostenerse que todos persiguen lo mismo, pues la diversidad de tendencias es reconducida por Aristóteles a este finalismo inmanente que lleva a cada especie a tratar de inmortalizarse en la medida de lo posible, y que caracteriza de "di-

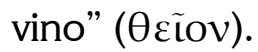

De entre todos los hombres, queda claro que para Aristóteles es el filósofo quien es capaz de reconocer esta divinidad que todas las cosas poseen naturalmente o, en otras palabras, el finalismo natural al que responden todas ellas. Como hemos visto, no solo el hombre sino también plantas y animales expresarían esta tendencia hacia la inmortalidad divina, solo que el hombre es quien más plenamente puede hacerlo en tanto puede percatarse de ella y llevarla a su máxima expresión. En varios pasajes del último libro de la Ethica Nicomachea aparece, respecto de la vida teorética, el calificativo de "divino" para la mejor de las vidas humanas ${ }^{35}$. Según ROMEYER (1983: 232), no hay contradicción, en pasajes como EN 1178b7, entre lo inmortal y lo humano

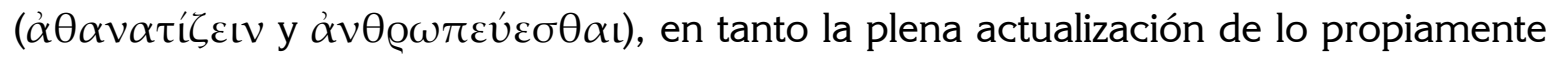
humano implica los modos de inmortalidad propios de la especie. En vistas de lo que he desarrollado a lo largo de este trabajo, Aristóteles sostiene una tendencia hacia lo divino que atraviesa todo el cosmos, que se verifica en cada individuo según las posibilidades de su especie, y que solo en el caso del hombre puede volverse un fin deliberado.

\section{La herencia platónica en la argumentación aristotélica acerca de la tendencia a la inmortalidad}

La construcción hermenéutica de una diferencia abismal e inconciliable entre las filosofías de Platón y Aristóteles puede actuar como un velo que impida al intérprete entender cómo se nutre un pensamiento filosófico de las enseñanzas de sus maestros y predecesores. A la hora de sopesar las influencias se debe escapar tanto del reduccionismo de atribuir idénticas tesis a autores diversos como de la construcción de antago- 
nismos irreconciliables. Este estudio sobre la apropiación aristotélica de uno de los argumentos platónicos sobre la inmortalidad busca aportar a esta discusión. La influencia del argumento platónico es innegable y abarca tanto los recursos argumentativos como la elección de la terminología de la participación y la comunicación. Aristóteles se vale de la $\mu \varepsilon \dot{\varepsilon} \theta \varepsilon \xi \iota$ y sus términos relacionados para expresar el finalismo no intencional, en un argumento que retoma los lineamientos principales de Symposium y Leges. Esta relación entre Symposium y Physica, poco estudiada en comparación con la relación de esta obra aristotélica con Phedon, muestra el marco polémico en el cual Aristóteles construyó su andamiaje causal. La influencia se extiende, como hemos visto, a otros pasajes donde Aristóteles argumenta acerca del impulso hacia la inmortalidad que poseen los animales, donde aparece el vocabulario de la $\mu$ í $ү \sigma \iota \varsigma$ y críticas análogas a las de Phedon a aquellos que no alcanzaron a una adecuada explicación de la causa final. Sin embargo, como he tratado de mostrar, no hay que llevar la interpretación mucho más allá, en tanto la lectura y las consecuencias de este cobran buen sentido apelando a conceptos básicos del pensamiento aristotélico. Esto, lejos de quitarle valor a la influencia del pensamiento platónico en la obra aristotélica, revela su carácter fructífero, en tanto mostraría que los argumentos de Platón son capaces de aportar elementos valiosos aun fuera de los límites del sistema teórico para el cual fueron pensados. En este juego de convergencias y divergencias, ambos encuentran que hay un orden divino

en el cosmos que devela su naturaleza finalista dentro y fuera de los límites de la intención ${ }^{36}$.

\section{Bibliografía}

\section{Ediciones y traducciones}

Barbotin, E. et Jannone, A. (1966) Aristote. De L'Ame, Paris, Les Belles Lettres.

Calvo Martínez, T. (1994) Acerca del alma, Madrid, Gredos.

DiÈs, A. (1956) Platon. OEuvres complètes, XII 1 et XII 2, Les Lois, Paris, Les Belles Lettres.

DOVER, K. (1980) Plato. The Symposium, greek text with commentary, Cambridge, University Press.

HamlyN, D. (1968) Aristotle. De Anima. Books II and III (with passages from book I). Oxford, Clarendon Press, 1968, 1993.

MoviA, G. (1979) Aristotele, L'Anima, trad., introd. y com., Napoli, L. Loffredo.

PECK, A. (1979) Aristotle. Generation of Animals, greek text with notes and commentary, London, Loeb.

Ross, W.D. (1961) Aristotelis De anima, recogn. brevique adnotatione instruxit, Oxford, Clarendon. 


\section{Bibliografía secundaria}

Aubenque, P. (1966) Le problème de l'être chez Aristote, Paris, Quadrige (citado por la trad. cast. De Vidal Peña, Madrid, Escolar y Mayo, 2008).

BALME, D. M. (1962) "Development of biology in Aristotle and Theophrastus: theory of spontaneous generation", Phronesis Vol. 7, No. 1, pp. 91-104.

BERTI, E. (2005) Nuovi studi aristotelici, Brescia, Morcelliana.

BERTI, E. (2008) Las razones de Aristóteles, Buenos Aires, Oinos.

BotTer, B. (2005) Dio e divino in Aristotele, Saint Augustin, Academia Verlag.

BOTTERI, G. y CASSAZA, R. (2015) El sistema astronómico de Aristóteles. Una interpretación, Buenos Aires, Biblioteca Nacional, 2015.

BROADIE, S. (1993) "Que fait le premier moteur d'Aristote? Sur la théologie du livre Lambda de la Métaphysique”, Philosophique de la France et de l'Étranger (183), pp. 375-411.

BURNYEAT, (2004) "Aristotle and the foundation of sublunary physics", en MANSFELD, J. AND DE HAAS, F. Aristotle. On Generation and Corruption, i. Proceeding of the 15th Symposium Aristotelicum, Oxford, University Press, pp. 7-24.

CLEARY, J. (1988) Aristotle on the many senses of priority, Carbondale, Illinois, Southern Illinois University Press.

COOPER, J. (1982) “Aristotle's natural teleology", en SCHOfiEld M. AND NussBaum, M. (eds.), Language and logos, Cambridge University Press, pp. 197-222.

CORNFORD, F. (1935) Plato's theory of knowledge, London, Routledge and Kegan Paul, 1935. Reprint, Indianapolis: Bobbs-Merrill, 1957.

Di CAMILlo, S. (2016) Eîdos: La teoría platónica de las ideas, La Plata, EDULP.

FALCON, A. (2005) Aristotle and the science of nature: Unity without Uniformity, Cambridge, University Press.

FUJISAWA, N. (1974) "Échein, metéchein, and idioms of 'paradeigmatism' in Plato's theory of forms", Phronesis, Vol. XIX, pp. 30-58.

FurLEY, D. (1985) “The rainfall example in Physics ii.8”, en GOTTHELF, A. (ed.), Aristotle on nature and living things, Mathesis, Pittsburgh and Bristol, pp. 177-82.

GAUTHIER, R. ET JOLIF, J. (1970) L'Éthique à Nicomaque. 2e édition avec une introduction nouvelle, Louvain, Publications universitaires.

Gerson, L. (2005) Aristotle and other Platonists, New York, Cornell University Press.

GotTHELF, A. (1987) "Aristotle's conception of final causality", en GOTTHELF, A. AND LENNOX, J. (eds.), Philosophical issues in Aristotle's biology, Cambridge, University Press, pp. 204 42. 
JAEGER, W. (1923) Aristoteles. Grundlegung einer geschichte seiner entwicklung, Berlin (citado según la traducción española de Gaos, J. Aristóteles, México D. F., Fondo de Cultura Económica, 1946.)

Johnson, M. (2005) Aristotle on teleology, Oxford, University Press.

Judson, L. (2005) "Aristotelian Teleology", Oxford Studies in Ancient Philosophy, vol. XXIX, Oxford, University Press, pp. 341-366.

KAHN, C. (1985) "The place of the prime mover in Aristotle's teleology", en Aristotle on Nature and Living Things, edited by A. GOTTHELF. Pittsburgh, Mathesis Publications, Inc. pp. 183206.

KLOSKO, G. (1986) The Development of Plato's political theory, New York, Methuen.

LEFÈVRE, C. (1972) Sur l'évolution d'Aristote en psychologie, Louvain, Éditions de l'Institut supérieur de philosophie de l'Université catholique de Louvain.

LLOYD, G. (1993) "L'Idée de nature dans la Politique d'Aristote", en AUBENQUE, P. Y TORDESILLAS, A. (eds.), Aristote Politique. Etudes sur la Politique d'Aristote, París, PUF, pp. 135-159.

MATTHEN, M. (2001), "The holistic presuppositions of Aristotle's Cosmology", Oxford Studies in Ancient Philosophy, vol. XX, Oxford, University Press, pp. 171-199.

Nussbaum, M. (1978) Aristotle's De motu animalium Princeton, University Press.

POLANSKY, R. (2007) Aristotle’s De Anima, Cambridge, University Press.

QuARANTOTTO, D. (2005) Causa finale, sostanza, essenza in Aristotele, Roma, Bibliopolis.

REALE, G. (2004) Eros, demonio mediador. El juego de las máscaras en el Symposium de Platón. Barcelona.

ROMEYER DHERBEY, G. (1983) Les choses mêmes. La pensée du réel chez Aristote, Lausanne, L'Age d'Homme.

Ross, A. (2016), HoRn, CH. (ed.), Aristotle's Metaphysics Lamba. New Essays, Boston/Berlin, De Gruyter, pp. 207-227.

Ross, D. (1924, 1948) Aristotle, Metaphysics, 2 vols., Oxford, Clarendon Press.

RossI, G. (2010) “Aristóteles y la lluvia, una vez más”, Diánoia LVI, pp. 91-123.

RowE, C. (2010) "The relationship of the Laws to other dialogues: A proposal", en BOBONICH, C. (ed.), Plato's Laws. A critical guide, Cambridge, University Press, pp. 29-50.

SABINE, G. (1961) A history of political theory, New York, Holt, Rinehart and Winston.

SEDLEY, D. (1991) “Is Aristotle's Teleology Anthropocentric?”, Phronesis 36, pp. 179-96.

SEIDL, H. (1995) “On the concept of God in Aristotle's De Philosophia”, L'Aristote perdu, Roma, CNR, pp. 69-90.

SOlANA DuESO, J. (2005) "La construcción de la diferencia sexual en Aristóteles", Convivium 18, pp. 23-46. 
WiElAnd, W. (1992) Die aristotelische Physik. Untersuchungen über die Grundlegung der Naturwissenschaft und die sprachlichen Bedingungen der Prinzipienforschung bei Aristoteles, Vandenhoeck \& Ruprecht, Göttingen.

\section{Notas}

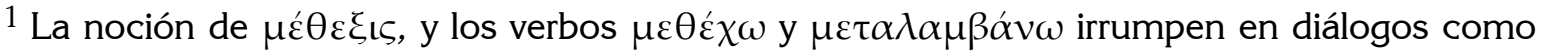
Phedon (100c4; 102b2) y Respublica (476d2-3) para explicar la relación entre los particulares sensibles y las Formas. En Parmenides es uno de los ejes centrales de las objeciones que el personaje homónimo le plantea a un joven Sócrates necesitado de entrenamiento dialéctico. En Sophista 252d-256a se desliza a la caracterización de una relación interna entre Formas.

2 Este uso, que podemos denominar "no técnico", aparece también en Platón. Cf., p.e., Resp.

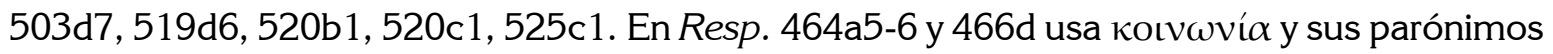
con un sentido análogo a $\mu \varepsilon \dot{\varepsilon} \theta \varepsilon \xi ı$. Acerca de la multiplicidad de sentidos de los términos que suelen ser traducidos por "participación", (cf. FUJISAWA 1974).

3 Ejemplos de estos tres usos caracterizan: 1) la participación de la especie en el género: Top. 121a10-19; 122a9; 143b14, 21; Metaph. 1037b19; 1059b33; 2) la relación del individuo con

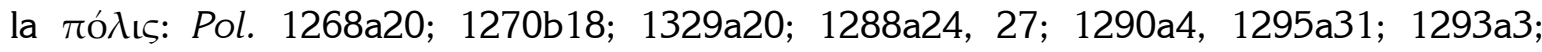
1306b10; 1340b36; 3) la crítica de la noción platónica: Metaph. 990b31; 991a1; 956b8; 990b28; 992a28; 1079a27; 1217b11; 1040a27; 1079a25.

4 QUARANTOTTO (2005:14) caracteriza de esta forma los usos de la causa final en aquellos casos donde, precisamente, no es posible atribuir al agente una intención. Simplicio, 250, 22-ss., si bien habla de la forma, dice que las entidades sensibles no solo dependen de la forma y se conservan por ella, sino que también se esfuerzan por alcanzarla, esto es, tienden a ella, aunque

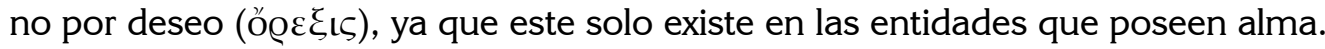

${ }^{5}$ Así, CORNFORD (1935:2) sostiene que "the immortality and divinity of the rational psyche, and the real existence of the objects of its knowledge -a world of intelligible Forms separate from the objects our senses perceive" as the "two pillars' of Platonism."

6 Diotima lo califica de gran divinidad ( $\pi \tilde{\alpha} v$ tò $\delta \alpha \mu$ óvıov, 202d12), intermedia entre lo divino y lo mortal ( $\mu \varepsilon \tau \alpha \xi \hat{v} \varepsilon \dot{\sigma} \sigma \iota$ $\theta \varepsilon \sigma \tilde{v} \tau \varepsilon \kappa \alpha i ̀ ~ \theta v \eta \tau o \tilde{v}, 202 \mathrm{e} 1$ ). Su función mediadora es cósmica: "estando en medio de ambos plenifica, de modo que el todo mismo queda unido consigo mismo" (202e). Reale (2004: 91-92) indica que no solo Eros es intermedio en cuanto a su naturaleza, sino que se mueve entre los dos ámbitos, posibilitando la comunicación entre los animales y los dioses.

7 Todas las traducciones del griego son propias.

8 "No es, por naturaleza, ni inmortal ni mortal", 203d-e.

${ }^{9}$ Así lo afirma en De an. I 1, 402b4-5, al sostener que los investigadores contemporáneos solo se ocupan del alma humana y dejan de lado la cuestión tanto del alma animal como divina.

10 El rol del Eros en el Symposium recuerda la función de los principios de Amor y Odio en la 
filosofía de Empédocles (ver, por ejemplo, el rol de la $\varphi$ ılí $\alpha$ en B16, B17, B18). Sin embargo, en el caso de Platón, no se trata de dos fuerzas opuestas sino de un solo principio.

11 Varios intérpretes han entendido el proyecto político de Leges como un intento de reformular el proyecto político del rey filósofo de Respublica para reemplazarlo por el gobierno del filósofo sobre la base de un código legislativo religioso (KLOSKO 1986: 198-99; SABINE 1961:75)

12 No en vano, en este texto platónico, "el dios sería la medida de todas las cosas" (Leges, IV, 716c4-5) y no el hombre, como sostienen algunos. El texto prosigue sin nombrar a Protágoras.

13 Al final del libro II de Ethica Nicomachea, Aristóteles reconoce la dificultad para alcanzar el término medio propio de la virtud, por lo cual afirma que "ser virtuoso es una tarea" (ع́ơov

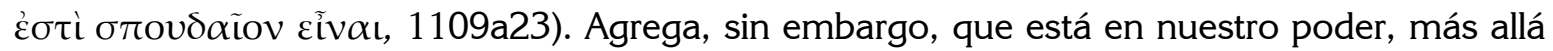

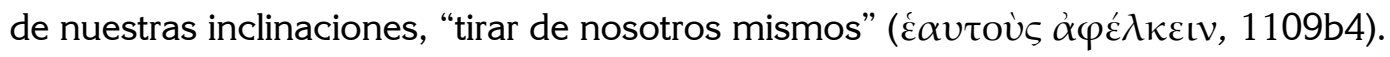

14 Para este autor, en los escritos prácticos aristotélicos, el aspecto normativo de la noción de naturaleza convive, en tensión, con un aspecto descriptivo.

15 En tanto el fin de cada especie reside en la actualización de las capacidades propias del tipo de forma que posee, la generación de un ser vivo de la misma especie está siempre incluida dentro de los fines propios. En el caso de la especie humana se dan dos peculiaridades. Una, la posibilidad de negarse a actualizar este fin, como se verá infra. Otra, la posibilidad de alcanzar la inmortalidad por medio del ejercicio del intelecto, que trataré solo de modo sucinto, en tanto excede los límites de este trabajo.

16 La disputa acerca del alcance de la teleología aristotélica excede el marco puntual del funcionamiento de los seres vivos. La subsistencia de estos, en efecto, requiere de otras condiciones que permiten la vida en la Tierra. Un caso clásico de esto último es la discusión en torno al ejemplo aristotélico de la lluvia en Physica II, 8, 198b16-21. Leen este pasaje de modo teleológico COOPER (1982), FURLEY (1985), SEDLEY (1991), mientras que autores como NuSSBAUM (1978) y GOTTHELF (1985) restringen la actividad finalista a los seres vivos y sus partes, citando como apoyo a su tesis Physica II, 8, 198b4-9. Cf., también, para una lectura no finalista del ejemplo de la lluvia, Rossi (2010), quien realiza, además, un somero estado de la cuestión.

17 En esta versión del argumento Aristóteles no hace uso del vocabulario de la participación, aunque unas líneas más adelante, en II 1, 732a12-13, afirma que los seres viven por participar

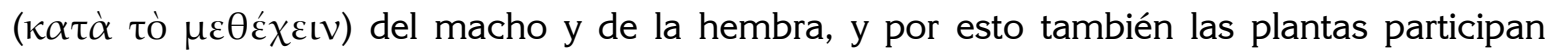
( $\mu \varepsilon \theta \varepsilon \dot{\chi} \chi \imath$ ) de la vida. Entiendo que se trata de un uso cercano a la participación de la especie en el género. Cf. nota 3.

18 El concepto de vida es central en el pensamiento aristotélico. En este texto, como en otros, la superioridad de la vida sobre el no vivir funciona como un principio. Cf., p.e., EN IX, 9, 1170a19-20 donde la vida aparece caracterizada como buena y placentera por sí misma y Metaph. XII, 7, 1072b 26-29. En este último pasaje Aristóteles afirma que el primer motor inmóvil está vivo, y posee una vida eterna y perfecta.

19 Esta concepción obedece al análisis que Aristóteles realiza del rol del macho y la hembra en 
el proceso de generación. El primero aportaría la forma y la segunda solo la materia. Entonces, la jerarquía macho/ hembra se funda en la que se verifica entre forma y materia (GA I 2, 716a6).

En Metaph. X 9, 1058b23-24, afirma que el mismo esperma da lugar a un macho o a una

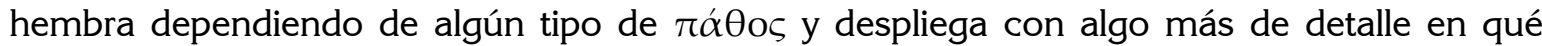
consistiría esta afección en GA I 20, 728a 18-21; II 1, 731b-732a y II 3, 737a25-29. Cf. el excelente estudio de SOLANA DUESO (2005), quien explica el establecimiento de este tipo de jerarquía biológica a partir del modelo aportado por la $\tau \varepsilon ́ \chi v \eta$.

20 Solo en este tipo de generación se produce una oủoí $\alpha$ numéricamente nueva. Sigo, en este sentido, a BERTI (2008: 61-62), quien, basándose, en parte, en WIELAND (1962, 1992), sostiene que es la metafísica aristotélica la que depende de la física y no a la inversa.

21 Las únicas excepciones a esta tendencia a la inmortalidad la constituyen los animales que se generan de modo espontáneo, porque no hay allí un ser vivo previo, y también los animales incompletos. Cf., HA II 1, 500a12; VII 1, 539a15-25; GA I 20, 728b10; III 1, 749a18; IV 1, 766a26; III 11 y BALME (1962).

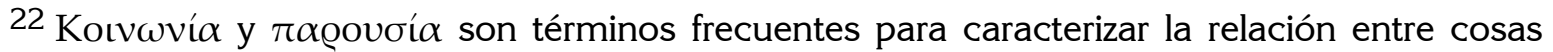
sensibles y Formas. Cf., p.e. Phd. 65d12-e1; 76d7-e4; 100b1-101c7; 102a10-105a5; Symp. 211b2-5; R. 476a4-7; c9-d3, 507b2-8, 596a5-597e5. Para otras referencias, ver Di CAMILLO (2016: 24).

23 Otros autores, como MOVIA (1979: 298) en su magistral comentario al De anima, se limitan a indicar la relación con Symposium y Leges en la misma lista de pasajes que incluye las otras apariciones en el Corpus aristotelicum.

24 Esta aclaración se repite en otros lugares del Corpus (Ph. II, 2, 194a33-36; Metaph. XII, 7, 1072b2-3; EE. VI, 15, 1249b15-16), si bien algunos pasajes como el de $P h$. II, 2 remiten al $D e$ phil., cuyo fragmento no se ha conservado.

25 Evidencia adicional puede encontrarse en Protrepticus, fr. 6 y 10c Ross; Phys., II 2, 194 a 35 y Metaph. XII 10, 1075a11-25. Cf. además SEIDL (1995).

26 Una postura diferente es defendida por MATTHEN (2001), quien entiende que todo el cosmos posee un fin único e interno a su propia dinámica. Cooper (1982) tiene una postura intermedia, en tanto cree que el fin en el ámbito sublunar es el mantenimiento de la vida en su conjunto, y no solo los seres humanos.

27 Me acerco así a la lectura de JOHNSON (2005). Respecto de la posibilidad de sostener la tesis fuerte de que esta relación entre especies suponga también una suerte de finalismo de segundo orden, cf. las críticas de Rossi (2010) a SEDLEY (1991).

${ }^{28}$ Según Metaph. XII 6, 1072a9-17, el movimiento del sol funciona como causa de la alternancia del nacimiento y la muerte (cf. GC 336b15).

29 Para poder entender este juego de prioridades es útil recurrir a los múltiples sentidos de prioridad identificados por CLEARY (1988: 75-85).

30 Remito al trabajo mencionado de Ross (2016) para un buen estado de la cuestión acerca de la posibilidad de encontrar en el primer motor inmóvil una causa formal, eficiente o final. 
31 Gran parte de la discusión sobre la causalidad final del primer motor se plantea acerca de su funcionamiento respecto de la primera esfera. Según KAHN (1985: 184-5), las lecturas más restringidas de Metaphysica XII sostienen que la relación de dependencia se da solo respecto del ámbito sublunar o, más estrechamente todavía, del primer cielo. En la versión más amplia, sustentada por el propio KAHN, toda tendencia a la actualización, sea en los ámbitos sub o supra lunar, dependen de la acción causal del primer motor inmóvil. Excede los límites de este trabajo la consideración de esta relación. Acerca de la explicación astronómica aristotélica, que toma al cosmos en su conjunto, cf. el excelente trabajo de BOTTERI Y CASSAZA (2015).

32 En Metaph. VII 7, 1032 a19 Aristóteles señala que todos los seres engendrados, por naturaleza o por arte, tienen materia y eso es lo que manifiesta su contingencia.

33 Tal es el argumento de Aristóteles en Metaph. XII 6, 1071b3. Si no existiera una entidad eterna, sino que todas fueran corruptibles, todas las cosas serían corruptibles (porque todo lo que es o bien es oúoí $\alpha$ o está relacionado con ella), incluso el movimiento y el tiempo, lo cual es absurdo.

34 Autores como AUBENQUE (1968: 281) extraen de esto una dualidad insuperable entre ontología y teología en el pensamiento aristotélico, al que llega a considerar incluso un antecedente del neoplatonismo respecto de la trascendencia y separación del primer principio.

35 Aparece también una caracterización análoga en Protrepticus 108 y 118 (DüRING) y De philosophia 8b y 8c (Ross).

36 Agradezco los comentarios y sugerencias del evaluador anónimo a la primera versión de este trabajo. 